$\$=$

\title{
Small bowel obstruction due to phytobezoar and adhesions following laparoscopic cholecystectomy
}

\author{
Nidal Abu Jkeim, Ahmad Al Hazmi, Awad Ali M. Alawad*, Rashid Ibrahim, \\ Ahmad Abu Damis, Samir Tawfik, Mohammed Mansour \\ Department of Surgery, Prince Sultan Armed Forces Hospital, Madina, Saudi Arabia \\ *Corresponding author E-mail:awadali82@hotmail.com
}

\begin{abstract}
We report a case of 51 -year-old female with history of laparoscopic cholecystectomy presented with abdominal pain and diagnosed as small bowel obstruction caused by adhesions. The initial presentation was periumbilical pain with nausea and vomiting. Plain abdominal radiograph showed dilated small bowel loops and multiple air fluid levels. Due to failure of conservative treatment, laparotomy was performed. An open metallic clip was adhering the bowel to the gallbladder fossa causing sharp angulation. A phytobezoar proximal to this angulation was exteriorized through enterotomy. The patient was recovered smoothly and discharged from our hospital.
\end{abstract}

Keywords: Metallic Clip Complications; Phytobezoar; Intestinal Obstruction; Adhesions

\section{Introduction}

Surgical clips have traditionally been considered inert and their loss in the peritoneal cavity is not accompanied by clinical consequences. The clips may slip due to incorrect application, or even during dissection, retraction, or aspiration (Stephens et al. 2010). When it is known during surgery that there are loose clips in the peritoneum, these are usually recovered laparoscopically without great difficulty, but there are occasions when, inadvertently, clips may remain in the peritoneal cavity. Complications of dropped or slipped clips are very rare. The most common complications are abscess formation and migration (Chong \& Chong 2010).

Small bowel obstruction is a common surgical emergent condition. The most common causes of small bowel obstruction are adhesion (73.8\%) and hernia (18.5\%) (Ezzat et al. 2009). Small bowel obstruction caused by bezoars is rare, and accounts for only 4\% (Robert et al. 2015). The word 'bezoar' derives from either the Arabic term 'badzehr' or the Persian word 'padzahr' both of which denote counter-poison or antidote (Pujar et al. 2013). This word was applied to a greenish, hard concretion found in the fourth stomach of the Syrian goat. The stone was felt to prevent poisoning and came to Europe as the bezoar-stone which was highly prized for its medicinal properties. There are four types of bezoars: phytobezoars, trichobezoars, pharmacobezoars, and lactobezoars. Phytobezoars are a concretion of poorly digested fruit and vegetable fibres that are found in the alimentary tract (Yakan et al. 2010). These usually take the form of orange pith or pulp in patients with a history of surgery, or persimmon in patients without previous surgery.We are reporting small bowel obstruction in a patient who had had laparoscopic cholecystectomy. The obstruction was caused by phytobezoar proximal to an ileal kink.

\section{Case report}

A 51-year-old woman was admitted to the Emergency Department with complaints of abdominal pain and vomiting for two days. The pain was colicky and located primarily in the periumbilical area. The vomit was bile-stained. There is abdominal distension. Her medical history included diabetes mellitus. She underwent laparoscopic cholecystectomy two years ago.

On physical examination, her blood pressure was $148 / 75 \mathrm{mmHg}$, pulse rate 114 beats/minute, body temperature $36.8^{\circ} \mathrm{C}$. On auscultation, her heart sounds were normal and lungs were clear. Abdominal examination showed a distended abdomen with tenderness, which was worse in the periumbilical area, without guarding or rebound tenderness. No masses were felt. Her hernia orifices were normal. Bowel sounds were exaggerated. Rectal examination found an empty rectum with no palpable mass.Other systemic examination was unremarkable.

The laboratory findings revealed; sodium of $142 \mathrm{mmol} / \mathrm{L}$; potassium of $3.5 \mathrm{mmol} / \mathrm{L}$, white blood cell count (WBC) $10.7 \times 103 / \mu \mathrm{L}$, Hemoglobin (Hb) $11.2 \mathrm{~g} / \mathrm{dL}$ and Platelets (Plt) 372x103/ $\mu \mathrm{L}$. Renal function tests were within normal limits. Plain abdominal X-ray revealed multiple air fluid levels with dilated small bowel loops, suggestive of small bowel obstruction (Fig.1).

A preliminary diagnosis of intestinal obstruction caused by adhesions was made. Immediate fluid resuscitation and intravenous antibiotics were initiated. Upon admission, the patient was placed on cefuroxime and metronidazole. Nasogastric intubation failed to relieve a progressive increase in abdominal distension. Foley catheter was inserted to measure the urine output. The patient had high blood glucose and high serum lactic acid.Urine analysis showed ketonuria and arterial blood gases showed ph of 7.19 and bicarbonate of $12 \mathrm{mmol} / \mathrm{l}$. Combination of both ketoacidosis, and lactic acidosis worsened the general condition to the patient. Treatment against acidosis was started. 
The patient was taken for emergency exploratory laparotomy. There were markedly dilated loops of small bowel down to the ileum, where the loop of bowel was densely adherent to gall bladder fossa. Adhesions were released, and two metallic clips (Fig. 2) were found at the antimesenteric border of the bowel, causing kinking. An intraluminal mass was felt at the site of kinking, which was found at a distance of $100 \mathrm{~cm}$ from the ileocecal junction. It was milked distally. Enterotomy was performed and revealed a mass made of undigested vegetable matter measuring $5 \times 3$ $\mathrm{cm}$ (Fig. 3). The enterotomy was closed in two layers. On hospital day \#2, patient was significantly improved with the softened ab- domen, improved leukocytosis, and increased urine output. On hospital day \#3, the patient continued to improve with decreased abdominal distention and pain in addition to passing flatus. Oral diet was given 24 hours after removing the nasogastric tube on the third postoperative day. She was placed on enoxaparin $40 \mathrm{mg}$ SQ every 24 hours for deep-vein thrombosis prophylaxis. On the ninth day of her hospitalization, the patient was discharged in good condition. She was seen four weeks postoperatively with no complaint.
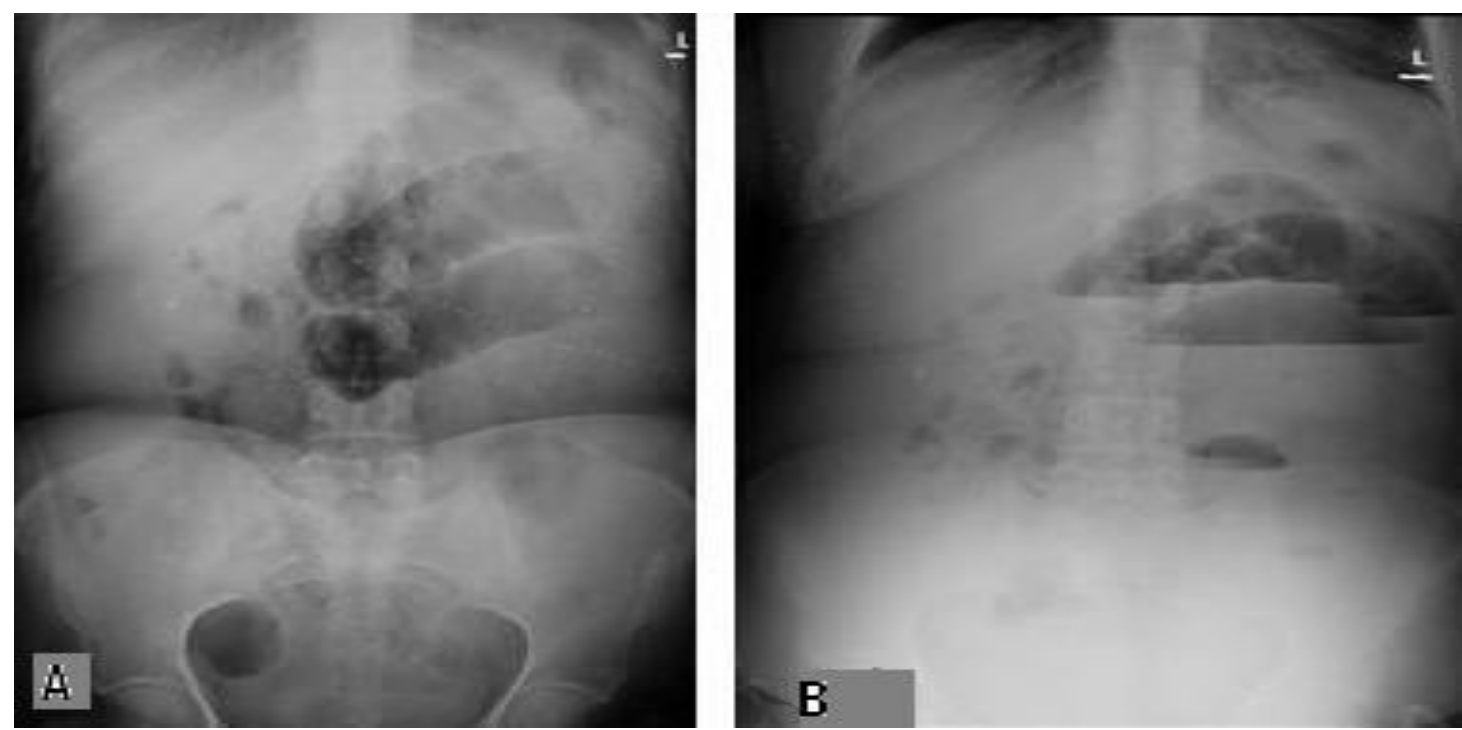

Fig. 1: Image Showing: A) Dilated Small Bowel Loops; B) Multiple Air Fluid Levels

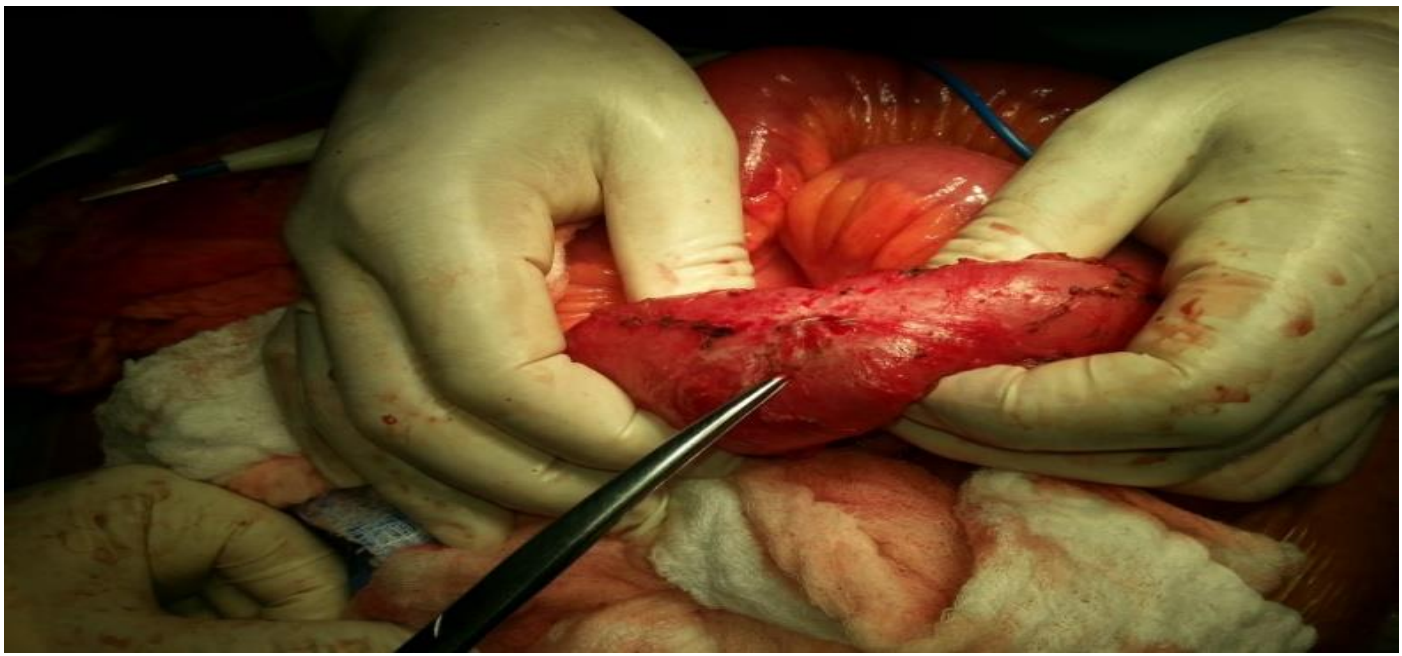

Fig. 2: Intraoperative Image Showing Site of Adhesions and the Metallic Clip on the Bowel Wall

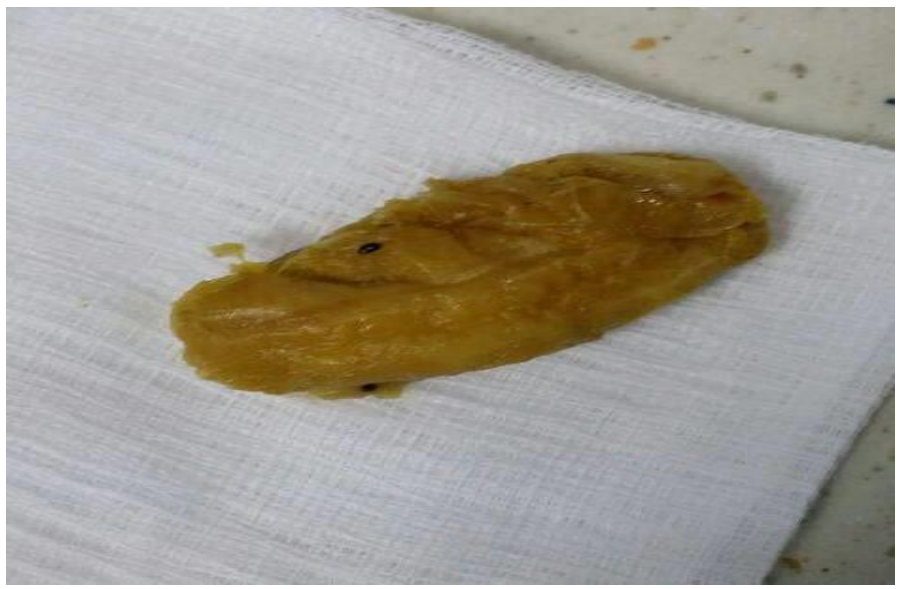

Fig. 3: Image Showing the Phytobezoars 


\section{Discussion}

There are no studies, which allow estimating the incidence of dropped clips in the peritoneal cavity during laparoscopic cholecystectomy. However, complications related to intra-abdominal clips have already been described. In a review from the literature, we have found four patients with prolonged abdominal pain related to clips in the peritoneum (Chong \& Chong 2010), one patient with chronic pelvic pain due to a clip lodged in the right ovary (Stephens et al. 2010), and another four patients with intraabdominal abscesses (2 with associated empyema) due to clips (Chong \& Chong 2010). Episodes of upper digestive bleeding due to a duodenal ulcer caused by a migrated clip following laparoscopic cholecystectomy have also been described (Hsin et al 2015). Except for the articles on the migration of clips to the common bile duct and the formation of calculi, all of the clinical cases describing lost clips have been published within the last seven years. Though only dealing with a very low number of complications, currently there are no studies that have studied the possible long-term consequences of lost or slipped clips, so the natural history of clips in the peritoneum should still be considered uncertain and not as innocuous and banal as initially believed. In our case, we thought that bowel adhesion causing sharp angulation is related to dropped metallic clips.

Primary small bowel bezoar is very rare and normally forms in patients with an underlying small bowel disease like diverticulum, stricture or tumor. Phytobezoar can also develop secondarily if there are areas of sufficient stagnation within a dilated bowel segment. This may occur in patients with strictures caused by Crohn's disease (Taylan et al. 2010), tuberculosis or previous surgery, or in patients with small bowel diverticula (Cantrell et al. 2014). In such cases, bile constituents or calcium salts contribute to bezoar development.

Small bowel obstructions account for $20 \%$ of hospital admissions Common causes are adhesions, strangulated hernia, malignancy, volvulus and inflammatory bowel disease. Phytobezoars are rare No particular age or sex prevalence has been observed. Bezoars can occur in any part of the gastrointestinal tract but are commonly reported in the stomach. Rarely, they occur in small intestines, colon, esophagus or common bile duct.

Bezoars of the small bowel are rare and are conceptualized to occur secondary to migration of a gastric bezoar. Most patients have a predisposing factor such as prior gastrointestinal surgery, particularly a previous gastrectomy which can facilitate transmigration. Diabetes mellitus, mixed connective tissue disease or hypothyroidism also, delay gastric emptying and cause bezoar formation (Tiwari et al. 2013). In our patient, long history of diabetes mellitus may contribute to this condition.

A plain radiograph typically shows a classic obstructive pattern. Occasionally we may be able to see the outline of a bezoar, which is actually difficult to differentiate from abscess or feces within the colon. This outline did not show in our patient's radiograph. Ultrasound has been used to detect bezoar. In a retrospective study done by Ripolles et al., ultrasound was able to detect phytobezoar in $88 \%$ of patients with small bowel obstructions (Yakan et al. 2010). A bezoar appears as a hyperechoic arc-like surface with acoustic shadowing on ultrasound; however, this feature may cause difficulty in differentiating bezoar from gallstones, which have similar ultrasound characteristics (Ezzat et al. 2009). In our case, gallstone ileus was excluded because of previous cholecystectomy.

Operative management entails laparotomy or resection anastomosis and is the main stay of treatment of small bowel bezoars. Alternatively, milking of small bowel bezoars into the caecum or stomach has been reported to circumvent the need for an enterotomy. In our patient, milking to either the stomach or the cecum was not possible due to adhesions. Only a few reports on laparoscopic removal have been published. The major difficulties for laparoscopic management are the size of bezoar and the presence of distended and fragile bowel loops (Parsi et al. 2013). Rarely, bezoars may be managed non-operatively. Coca Cola dissolution therapy for gastric bezoars, endoscopic removal with or without fragmentation in esophageal, gastric, duodenal bezoars and use of small bowel enemas for small bowel bezoars are the reported methods of non-operative management (Teicher et al. 2008).

Here, we described an unusual case of combined adhesive intestinal obstruction and phytobezoar. Kinking of the bowel secondary to previous laparoscopic cholecystectomy, as in our case, is very rare predisposing factor. This kinking was brought by dense adhesions at the gallbladder fossa, which can be explained by the presence of two metallic clips at the bowel wall. Surgical clips are thought to be the cause of adhesions and kinking of the bowel and its adherence to the gallbladder. They have traditionally been considered inert, and their loss in the peritoneal cavity is usually not accompanied by clinical consequences. To the best of our knowledge, this is the first case reported throughout the literature describing such as condition.

\section{Conclusion}

To summarize, small bowel obstruction from a phytobezoar is rare and often overlooked. We experienced a very rare condition of small bowel obstruction due to phytobezoar and adhesions caused by metallic clips. Although easily managed, early recognition with typical symptom and image findings play an important role in minimizing the morbidity and mortality.

\section{References}

[1] Cantrell EF, Cohen-Shohet RN, Browning RL, Richards WT \&Lottenberg L (2014): Small bowel obstruction secondary to impacted phytobezoar within a Meckel's diverticulum. Am Surg 80, e194-195.

[2] Chong VH \& Chong CF (2010): Biliary complications secondary to post-cholecystectomy clip migration: a review of 69 cases. J GastrointestSurg 14, 688-696. http://dx.doi.org/10.1007/s11605-009-1131-0.

[3] Ezzat RF, Rashid SA, Rashid AT, Abdullah KM \& Ahmed SM (2009): Small intestinal obstruction due to phytobezoar: a case report. $J$ Med Case Rep 3, 9312. http://dx.doi.org/10.1186/1752-1947-3-9312.

[4] Hsin MC, Lin IC \& Tai CM (2015): Duodenal ulcer caused by a surgical clip after laparoscopic cholecystectomy. Endoscopy 47 Suppl 1, E264. http://dx.doi.org/10.1055/s-0034-1391869.

[5] Parsi S, Rivera C, Vargas J \& Silberstein MW (2013): Laparoscopicassisted extirpation of a phytobezoar causing small bowel obstruction after Roux-en-Y laparoscopic gastric bypass. Am Surg 79, E93-95.

[6] Pujar KA, Pai AS \&Hiremath VB (2013): Phytobezoar: a rare cause of small bowel obstruction. J ClinDiagn Res 7, 2298-2299. http://dx.doi.org/10.7860/jcdr/2013/7248.3504.

[7] Robert B, Dhahri A, Rebibo L, Chivot C, Regimbeau JM \&Yzet T (2015): [Phytobezoar, an unusual cause of small bowel obstruction]. Presse Med 44, 476-477. http://dx.doi.org/10.1016/j.lpm.2014.06.020.

[8] Stephens M, Ruddle a \& Young WT (2010): An unusual complication of a dropped clip during laparoscopic cholecystectomy. SurgLaparo$\begin{array}{llll}\text { scEndoscPercutan Tech } & \text { 20, } 103-104 .\end{array}$ http://dx.doi.org/10.1097/SLE.0b013e3181df9b56

[9] Taylan SO, Mehmet G, Nihat Z \&Oguzhan I (2010): Small bowel obstruction by phytobezoar in Crohn's disease: strictureplasty. Am Surg 76, 13-14

[10]Teicher EJ, Cesanek PB \&Dangleben D (2008): Small-bowel obstruction caused by phytobezoar. Am Surg 74, 136-137.

[11]Tiwari A, Gupta V, Hazrah P, Chaudhary AK \& Lal R (2013): Phytobezoar: a rare cause of acute small bowel obstruction in an innocent abdomen. Arq Bras Cir Dig 26, 342-343. http://dx.doi.org/10.1590/S0102-67202013000400020.

[12] Yakan S, Sirinocak A, Telciler KE, Tekeli MT \&Denecli AG (2010): A rare cause of acute abdomen: small bowel obstruction due to phytobezoar. Ulus TravmaAcilCerrahiDerg 16, 459-463. 